\title{
Métricas de Avaliação de Algoritmos de Otimização
}

\author{
Miscelânia Pedrosa de Araújo $\quad$ Emerson Alexandre de Oliveira Lima \\ Programa de Pós-Graduação em Engenharia de Sistemas, PPGES-UPE \\ Escola Politécnica da Universidade de Pernambuco \\ Rua Benfica, 455 - Madalena - Recife/PE CEP: 50720-001 \\ E-mail: misspedrosa@gmail.com, eal@poli.br,
}

\section{RESUMO}

A área de Otimização trata do estudo de problemas de maximização ou minimização de uma função através de um conjunto de valores viáveis, em um determinado domínio. Os problemas de otimização buscam alcançar a solução ideal para um determinado problema, de forma que possa alcançar com o menor custo e/ou máxima eficiência os objetivos que foram estabelecidos[1]. Para isso, existem diversos algoritmos na literatura que podem ser utilizados para a solução de problemas de otimização [2].

Não há, entretanto, técnica de otimização que seja aplicável a toda sorte de problemas que ocorrem na prática. Ou seja, um algoritmo pode ser capaz de solucionar um determinado problema e não ter a mesma capacidade para solucionar outro, não havendo nenhuma forma que garanta encontrar o ponto ótimo global.

Desta forma, as últimas décadas tem presenciado um crescimento sem precedentes na criação e aplicação de novos algoritmos e heurísticas para solução de problemas de otimização. Atualmente, a comparação de eficiência entre essas diferentes heurísticas é feita mediante a aplicação de benchmarks $[1,3,7]$. Entretanto, tal comparação tem sido feita de forma relativamente livre não havendo um padrão ainda estabelecido de comparação [4].

Analisar a eficiência de algoritmos de otimização é de grande importância tanto na área acadêmica quanto na área aplicada. Geralmente, a comparação é realizada com um conjunto de algoritmos de forma a se selecionar o que obteve melhor desempenho na solução de uma categoria previamente escolhida de problemas [5]. O desempenho do algoritmo depende de vários fatores, não somente de sua complexidade teórica. Para cada conjunto problema-algoritmo é possível medir o tempo computacional, o número de falhas, o erro da solução obtida, entre muitos outros, e analisar esses resultados não é uma tarefa fácil. A quantidade de dados obtidos pode ser enorme o que pode dificultar a apresentação e interpretação dos resultados. Com isto, é proposto a utilização de performance profiles, uma técnica de comparação de diferentes algoritmos, que combina as melhores características para avaliação de desempenho [6, 2].

A proposta deste trabalho, desenvolvido como dissertação de mestrado, é estabelecer uma metodologia baseada no conceito de performance profiles e aplicar tal metodologia na comparação de diferentes estratégias heurísticas de otimização baseada em inteligência computacional em diversos benchmarks $[3,7]$.

A proposta original da métrica é, dado $S=\left\{s_{1}, s_{2}, \cdots, s_{m}\right\}$ conjunto de algoritmos testados na resolução de um conjunto $P=\left\{p_{1}, p_{2}, \cdots, p_{n}\right\}$ de problemas, definir $t_{p, s}$ como o tempo para o algoritmo $s \in S$ resolver o problema $p \in P . r_{p, s}=\frac{t_{p, s}}{\min _{s} \in t_{p, s}}$ é definido como o fator de eficiência do algoritmo $s$ na resolução do problema $p . \rho_{s}(\tau)=\frac{1}{m}\left|\left\{p \in P: r_{p, s} \leq \tau\right\}\right|$ é a probabilidade de solução de um problema pelo algoritmo $s$ com fator de eficiência menor ou igual a $\tau$. A métrica é definida como $\rho^{*}=\lim _{\tau \rightarrow \infty} \rho_{s}(\tau)$ mede a probabilidade da solução dos problemas de $P$ pelo algoritmo $s$ e a comparação dos algoritmos é feita pelo valor de $\rho_{s}$.

Resultados preliminares do primeiro ano desta pesquisa mostraram que o conceito original de performance profiles aplicado a algoritmos de inteligência computacional (inteligência de enxame, algoritmos genéticos, algoritmos meméticos, dentre outros) implicam em pouca informação acerca da eficiência 
relativa destes algoritmos. Modificações propostas na métrica original introduzindo termos relativos a proximidade da solução encontrada com a solução conhecida e número de operações (produtos, adições e comparações) necessárias forneceram informações adicionais que permitiram tal comparação.

Propostas de trabalhos futuros incluem teste da métrica proposta em solvers públicos (gsl, nag, etc) aplicados na solução de instâncias de problemas benchmarks em problemas de corte e caixeiro viajante.

Palavras-chave: Performance Profiles, Métricas Algoritmos, Otimização

\section{Referências}

[1] Barbosa, H. J., Bernardino, H. S., Barreto, A. M. (2013). Using Performance Profiles for the Analysis and Design of Benchmark Experiments. In Advances in Metaheuristics (pp. 21-36). Springer New York.

[2] Dolan, E. D., Moré, J. J., Munson, T. S. (2006). Optimality measures for performance profiles. SIAM Journal on Optimization, 16(3), 891-909.

[3] Dolan, E. D., More, J. J. (2002). Benchmarking optimization software with performance profiles. Mathematical programming, 91(2), 201-213.

[4] Hansen, N., Auger, A., Ros, R., Finck, S., Posik, P. (2010, July). Comparing results of 31 algorithms from the black-box optimization benchmarking BBOB-2009. In Proceedings of the 12th annual conference companion on Genetic and evolutionary computation (pp. 1689-1696). ACM.

[5] Martinez, J. M., Santos, S. A. (1995). Métodos computacionais de otimização. Colóquio Brasileiro de Matemática 20.

[6] Metz, E., Lencevicius, R. (2003). Efficient instrumentation for performance profiling. arXiv preprint cs/0307058.

[7] Moré, J. J., Wild, S. M. (2009). Benchmarking derivative-free optimization algorithms. SIAM Journal on Optimization, 20(1), 172-191.

[8] Weise, T. (2009). Global optimization algorithms-theory and application. Self-Published,. 